\title{
Carabamazepine and Cushing's: a cautionary tale of assay interference mimicking disease
}

Mie Mie Tisdale, Francesca Swords

Department of Diabetes and Endocrinology, Norfolk and Norwich University Hospital NHS trust, Norwich, UK

\section{Case studies}

○ Male, 44 years old

$\circ$ Presented to nephrology with malignant hypertension.

○ Routine investigation for secondary causes performed.

- Marked hyper-reninaemia, marked elevation in urinary free cortisol (UFC) and marked disparity of kidney size all present.

$\circ$ Referred to endocrinology for investigation of Cushing's syndrome but clinically no convincing evidence of Cushing's.

\section{○ PMH: bipolar disorder}

- DH: venlafaxine, carbamazepine, pericyazine, amlodipine $10 \mathrm{mg}$ daily, perindopril $4 \mathrm{mg} B D$, bisoprolol $5 \mathrm{mg}$ daily, spironolactone $100 \mathrm{mg}$ daily and phenoxybenzamine $10 \mathrm{mg} B D$.
- Female, 68 years old

Initial presentation to nephrologist with hypertension and obesity.

Routine investigation for secondary causes performed.

Elevated urinary free cortisol and lack of suppression after $1 \mathrm{mg}$ overnight dexamethasone test.

$\circ$ Referred to endocrinology for investigation of Cushing's but again unconvincing clinical assessment.

PMH: mental health issues

DH: venlafaxine, carbamazepine, amlodipine $10 \mathrm{mg}$ daily, ramipril $10 \mathrm{mg}$ daily, atenolol $50 \mathrm{mg}$ daily and atorvastatin $20 \mathrm{mg}$ at night.

\section{Examination findings}

\section{BP 150/110}

○ Slim (Height $184 \mathrm{~cm}$, Weight 78.7 kg, BMI 23.2)

$\circ$ Normal skin character, no bruising, striae, plethora or myopathy

\section{BP 170/84}

$\circ$ Obese (Height 158cm, Weight 78.1 kg, BMI 31.3)

Normal skin character, few pale striae and one bruise, no myopathy

\section{Investigations}

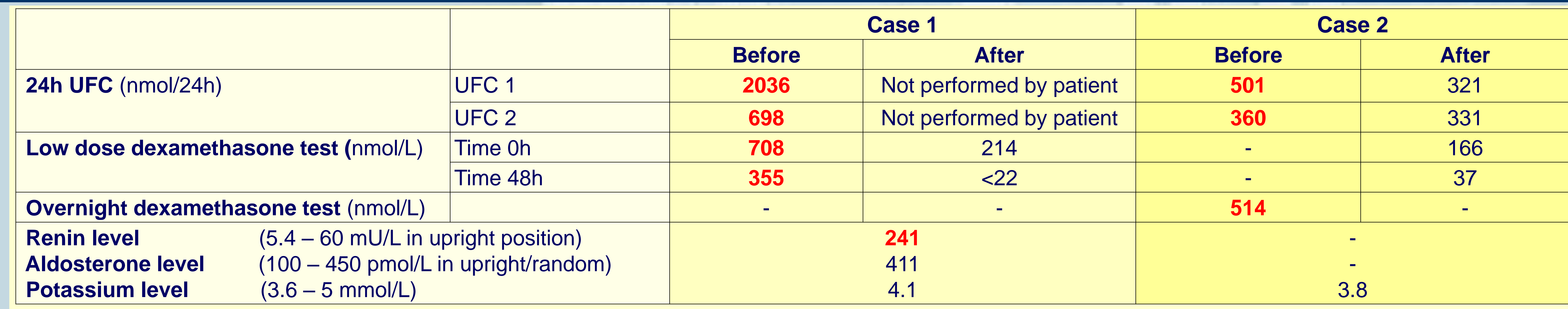

Potassium level $\quad(3.6-5 \mathrm{mmol} / \mathrm{L})$

Case 1: Before \& after carbamazepine withdrawal. (note: Case 1 had normal bone density and HbA1c)

Case 2: Before \& after changing serum cortisol assay from Roche Cobas assay to Abbott platform assay.(note: Case 2 remains on carbamazepine)

\section{Conclusions}

Both cases presented with hypertension but no obvious clinical features of Cushing's.

In both cases a non specialist performed screening test for Cushing's which were apparently positive.

Detailed clinical assessment by an endocrinologist refuted the diagnosis in both cases and considered carbamazepine interference either through the enzyme induction effects leading to false positive dexamethasone suppression test results, or by direct interference in some cortisol assays.

$\circ$ Drug withdrawal in one, and the use of an alternative platform in the other allowed the diagnosis to be excluded and prevented further unnecessary investigation and anxiety. 